\title{
Cleft treatment: A timely approach
}

\author{
Priti Shukla \\ Senior Resident, Dept. of Orthodontics and Dentofacial Orthopaedics, Purvanchal Dental College, Institute of Dental Sciences, \\ Gorakhpur, Uttar Pradesh, India \\ *Corresponding Author: \\ Email: drpritipankaj@gmail.com
}

\begin{abstract}
This article presents the case of a patient with unilateral cleft lip and palate, treated by dento-facial orthopedics followed by orthodontic intervention. The patient reported in the mixed dentition stage with repaired unilateral cleft lip and palate. Extraorally, the patient had a concave profile, obtuse nasolabial angle and negative lip step. Intraorally, there was an evident anterior crossbite, rotated central incisors and missing lateral incisors. The maxillary deficiency was addressed using facemask followed by orthodontic treatment to correct the dentoalveolar retrusion.
\end{abstract}

Keywords: Cleft palate, Facemask, Orthopaedics and Cleft.

\section{Introduction}

Cleft lip and/or palate are the most frequent craniofacial anomalies ${ }^{1}$ seen in human beings with an incidence of 1:800 live births. Isolated cleft palate occurs in 1: 2000 live births. Cleft occurs due to nonfusion or lack of fusion between the two halves of the palatal shelves. ${ }^{2}$ The etiologic factors can be classified as genetic or environmental. The cleft can be syndromic (as seen in Van Der Woude's syndrome) ${ }^{3}$ or nonsyndromic. Dental abnormalities, malformation, abnormal eruption pattern, deficient midface development, severe maxillary transverse deficiency and alveolar cleft can be seen in patients with CLP. ${ }^{4}$ The management of children with cleft lip and palate presents many challenges but also many rewards. Our involvement with these children and their families often begins before birth and can extend into late adulthood. Affected individuals present a multiplicity of problems and effective management involves a wide range of specialists. $^{5}$ In order to improve facial profile orthopedic and orthodontic intervention is necessary. The purpose of this case report is to present the effect of orthopedic treatment on the profile changes of a mixed dentition patient with unilateral cleft lip and palate.

\section{Case Report}

A male patient (aged 10yrs) reported to the department with a chief complaint of un-esthetic appearance which led to teasing by the peer group. The parents gave a history of unilateral cleft lip and palate which was repaired when the patient was 3.5yrs of age. No other local or systemic illness was reported.

On examination, the extraoral features revealed a concave profile, lack of nasal prominence, obtuse nasolabial angle and negative lip step. Frontally, he presented with flattened nasal bridge, depressed alar base of left side and a surgical scar running from the alar base to the upper lip on the left side. Intraorally, there was a cross bite extending from the deciduous second molar of one side to the other involving the rotated maxillary permanent central incisors. (Fig. 1) However, the maxillary permanent first molars were in normal relationship to their mandibular counterparts. Radiographically, orthopantamogram showed absence of permanent lateral incisors.

The treatment was carried out in two phases:

Phase I consisted of the orthopedic phase to address the maxillary deficiency by protracting the maxilla. Acrylic posterior bite planes with stainless steel hooks in the deciduous canine- molar area was fabricated for attachments of extra oral elastics (4 oz initially followed by $8 \mathrm{oz}$ later) and cemented on both sides of the upper arch. A Petit facemask was delivered to the patient.

A force of $400 \mathrm{gm}$ on each side was applied for 12 hrs daily till a positive overjet was achieved.

After a period of 8 months following maxillary protraction, there was an obvious improvement in the facial profile; correction of anterior crossbite; negative lip step was eliminated and a better nasolabial angle was observed.

Phase II consisted of orthodontic phase which aimed at the correction of the incisor rotations and maintain the achieved positive overbite and overjet. The permanent central incisors were bonded and a segmental 0.014" NiTi was ligated to them. After the correction of rotation the deciduous canines were also bonded and a 0.014" stainless steel (A.J.Wilcock) was ligated to this segment for aligning the anteriors. After this the permanent molars were banded and a 0.016 " $\mathrm{x}$ 0.022 " TMA wire was fabricated with a top crimped at the molar tube such that it holds the wire $2 \mathrm{~mm}$ in front of the incisors. This will allow for the proclination of incisor and increase in arch length.

OPG findings at this stage revealed both the maxillary canines in the erupting stage. Secondary bone grafting was thus considered to provide good bone support for the erupting left canine. For further investigations prior to grafting, a CBCT was done 
which revealed a $20 \mathrm{~mm}^{3}$ volume of bone defect present just distal to the left central incisor. Furthermore the eruption path of the canine was away from the defect area and also the volume of defect was too small to be considered for bone grafting. Due to these reasons secondary bone grafting was not deemed necessary at this stage.
The patient is now in the retention stage maintaining the corrections achieved and is under regular follow up. Further orthodontic treatment will be carried out once the permanent dentition is complete.

The early orthopedic orthodontic intervention has had a positive psychological impact on the patient self esteem and confidence.
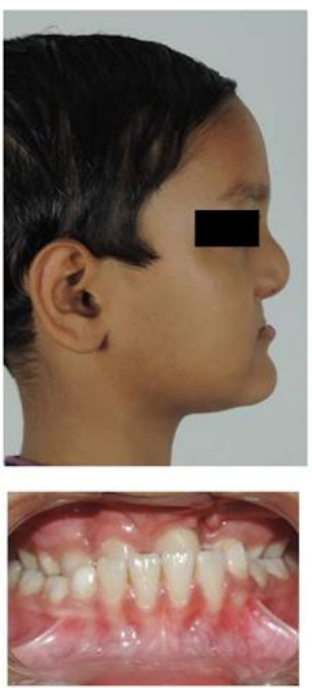
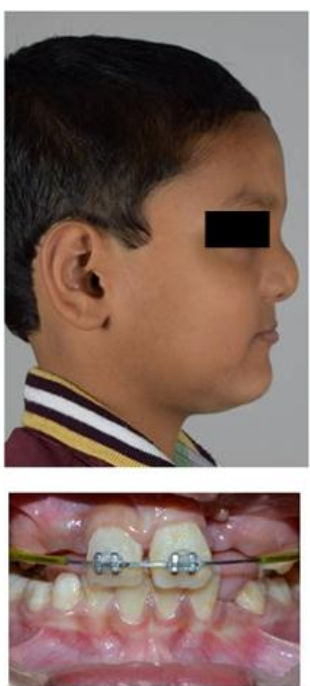

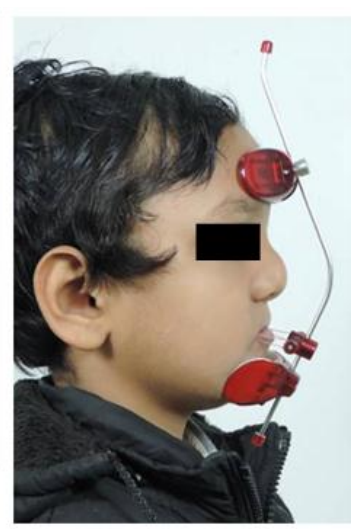

1a) Pre-treatment Extraoral b) Post-treatment Extraoral c) Pre-treatment Intraoral d) Post-treatment Intraoral e) with facemask
Fig. 1:

\section{Discussion}

True success in management of cleft lip\& cleft palate throughout an individual life can be ensured by the adherence of fundamental principles and successful management in a transdisciplinary approach.ie "Shared management "among all professional.

In orthopaedic intervention an attempt is made to influence the morphology of craniofacial skeleton. When extra-oral force is applied, the following appears in sequence:

1. An opening of the suture

2. Stretching of sutural connective tis sue

3. New bone deposition along the stretched fibres

4. Homeostasis which maintains the sutural width.

Early maxillary protraction has been recommended to treat midface retrusion in cleft lip and palate patients. Several clinical studies have shown favorable effects on the maxillary forward growth when the treatment was started at the deciduous or early mixed dentition stages (Irie and Nakamura, 1975; Friede and Lennartsson, 1981; Rygh and Tindlund, 1982; Ranta, 1988; Ishikawa et al., 1996). Because circummaxillary sutural growth is active during the early juvenile period (Scott, 1956; Bjo rk, 1966), it seems reasonable and advantageous to facilitate the forward growth of the maxilla at these stages. $^{6}$

The thing that encouraged us the most was a tremendous improvement in the confidence of the child and his self esteem.

\section{References}

1. Raghvan R, Sindhu S S, Kharanda O P. Craniofacial patterns of parents of children having cleft lip and/ or cleft palate anomaly. Angle Orthod 1994;64(2):137-144.

2. Atik E and Kocadereli I. Orthodontic Treatment for Cleft Palate Associated Open-Bite Malocclusion Using Compensatory Extraction Protocol: A Case Report. Research \& Reviews: Journal of Dental Sciences 2016;4:12-16.

3. Agarwal S, Dinesh MR, Dharma RM, Amarnath BC. Van der Woude Syndrome: management in the mixed dentition. Contemp Clin Dent 2013;4:105-7.

4. Lai MC, King NM, Wong HM. Abnormalities of maxillary anterior teeth in Chinese children with cleft lip and palate. Cleft Palate Craniofac J. 2009;46:58-64.

5. Hodgkinson P D, Brown S, Duncan D, Grant C, McNaughton A, Thomas P, Mattick C R. Management of children with cleft lip and Palate: a review describing the application Of multidisciplinary team working in this Condition based upon the experiences Of a regional cleft lip and palate centre in the United Kingdom: Fetal and Maternal Medicine Review 2005;16:1 1-27.

6. Ishikawa H, Kitazawa S, Iwasaki H, Nakamura S. Maxillary Protraction Combined With Chin-Cap Therapy: Cleft Palate-Craniofacial Journal, January 2000, Vol. 37 No. 1. 